INPLASY

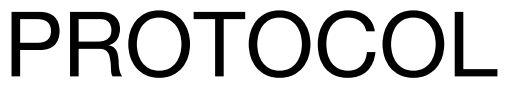

To cite: Feng et al.

Effectiveness of Tai Chi for Chronic obstructive Pulmonary

Disease: Overview of

Systematic Reviews and Metaanalyses. Inplasy protocol

2020100114. doi:

10.37766/inplasy2020.10.0114

Received: 27 October 2020

Published: 28 October 2020

Corresponding author:

Jiansheng Li

li_js8@163.com

Author Affiliation:

Henan University of Chinese

Medicine

Support: Key program of NNSF.

Review Stage at time of this submission: Preliminary searches.

Conflicts of interest:

There are no conflicts of interest.

\section{Effectiveness of Tai Chi for Chronic obstructive Pulmonary Disease: Overview of Systematic Reviews and Meta-analyses}

Feng, ZZ1; Li, XL2; Xie, Y3; Li, JS4.

Review question / Objective: What are the effects of Tai Chi for chronic obstructive pulmonary disease (COPD)? Many systematic reviews/meta-analyses have been conducted for Tai Chi's efficacy, but existed reviews summarized the evidence varied in their scope, quality, intervention time, control measures and reported outcomes, making the interpretation of the evidence complexed for clinicians and policy makers. In view of this, we plan to undertake an overview of reviews to evaluate the methodological quality and evidence quality of existed reviews and to provide comprehensive evidence of Tai $\mathrm{Chi}$ for COPD by resynthesized meta-analysis.

INPLASY registration number: This protocol was registered with the International Platform of Registered Systematic Review and Meta-Analysis Protocols (INPLASY) on 28 October 2020 and was last updated on 28 October 2020 (registration number INPLASY2020100114).

\section{INTRODUCTION}

Review question / Objective: What are the effects of Tai Chi for chronic obstructive pulmonary disease (COPD)? Many systematic reviews/meta-analyses have been conducted for Tai Chi's efficacy, but existed reviews summarized the evidence varied in their scope, quality, intervention time, control measures and reported outcomes, making the interpretation of the evidence complexed for clinicians and policy makers. In view of this, we plan to undertake an overview of reviews to 
evaluate the methodological quality and evidence quality of existed reviews and to provide comprehensive evidence of Tai Chi for COPD by re-synthesized meta-analysis.

Condition being studied: Tai Chi, a form of Chinese mind-body exercise, was a part of WHO's guide for action: Informal and selfdirected care, and performed as a complementary and alternative adjunct to pulmonary rehabilitation. Chronic obstructive pulmonary disease (COPD) is a worldwide public health challenge because of its high prevalence, mortality and disability, leading to significant economic and social burden. Pulmonary rehabilitation had been demonstrated to reduce dyspnea, increase exercise capacity, and improve quality of life in individuals with COPD. Many systematic reviews (SRs) /metaanalyses (MAs) have been conducted to prove the effects of Tai Chi for COPD during the past several years. High-quality SRs/MAs can provide a reliable scientific basis for clinicians, patients, and other decision makers. However, existed SRs/ MAs showed varied and heterogeneous results. To summarize the evidence on the effects of Tai Chi for COPD, we plan to conduct an overview of existing SRs/MAs.

\section{METHODS}

Search strategy: The international electronic databases of Pubmed, Embase, Cochrane library, and Web of science and Chinese databases of China National Knowledge Infrastructure Database (CNKI), WANFANG DATA, Chinese Biomedical Literature Database (CBM) and Chongqing VIP (CQVIP) were searched without language restriction. Detailed search strategies were developed for each database for title or abstract related to "Tai Chi” (as well as "Taiji”, "Taijiquan", "Tai Chi Chuan", et al), merging "chronic obstructive pulmonary disease" (as well as "COPD", "chronic obstructive lung disease", "Chronic Airflow Obstruction", et al). Meanwhile, we reviewed the references of included literatures.
Participant or population: Patients who are diagnosed with COPD by standard diagnostic criteria.

Intervention: Tai Chi (any form of Tai Chi, such as 24-style Tai Chi, Chen-style Tai Chi, Yang-style Tai Chi, et al), with or without usual care (routine medical treatment).

Comparator: Usual care or any other exercise (such as breathing exercise, walking exercise, aerobic exercise, et al).

Study designs to be included: Systematic reviews/meta-analyses of randomized controlled trials.

Eligibility criteria: 1) Patients who were diagnosed with COPD by standard diagnostic criteria; 2) The intervention groups were Tai Chi (any form of Tai Chi, such as 24-style Tai Chi, Chen-style Tai Chi, Yang-style Tai Chi, et al), with or without usual care (routine medical treatment); 3) The control groups were usual care or any other exercise (such as breathing exercise, walking exercise, aerobic exercise, et al); 4) At least one outcome followed was measured: exercise capacity [measured by 6-minute walking distance (6MWD)], quality of life [measured by St. George's Respiratory Questionnaire (SGRQ)], lung function[measured by forced expiratory volume in 1 second (FEV1) or FEV1 percent predicted normal values (FEV1\%)]; 5) SRs/ MAs of randomized controlled trials (RCTs).

Information sources: We review the references of included literatures to avoid the occurrence omission. Also, we will contact with author in case that we could not obtain full text.

Main outcome(s): Exercise capacity [measured by 6-minute walking distance (6MWD)], quality of life [measured by St. George's Respiratory Questionnaire (SGRQ).

Additional out come (s): I ung function[measured by forced expiratory volume in 1 second (FEV1) or FEV1 percent predicted normal values (FEV1\%)]. 
Quality assessment / Risk of bias analysis: The methodological quality of included SRs/MAs will be assessed by the Assessment of Multiple Systematic Reviews 2 (AMSTAR2). The quality of evidence for outcomes of FEV1, FEV1\%, 6MWD and SGRQ will be assessed using the Grading of Recommendations, Assessment, Development and Evaluation (GRADE).

Strategy of data synthesis: We re-analyze outcomes by RevMan software (version 5.3; The Cochrane Collaboration, Oxford, UK). Data is presented as the mean and standard deviation. The effect size is estimated by mean difference (MD) with 95\% confidence intervals (Cl) of change score for all outcome measures are continuous. A fixed effect model will be used if there is no statistically significant heterogeneity, or a random effect model is employed. The $\chi 2$ test with $\mathrm{p} 50 \%$ indicates significant heterogeneity.

Subgroup analysis: Subgroup analysis will be conducted based on the duration of Tai Chi: short period time ( $\leq 3$ months) and long period time ( $>3$ months).

Sensibility analysis: We will conduct sensibility analysis if it is necessary.

Language: There is no language limits on the search.

Country(ies) involved: China.

Keywords: Tai Chi; Overview; Chronic obstructive pulmonary disease; Systematic review; Meta-analysis.

Contributions of each author:

Author 1 - Zhenzhen Feng search the literature, conduct the statistical analysis and draft the manuscript.

Author 2 - Xuanlin Li screen the studies, extract the data, and evaluate the methodological quality of included systematic reviews/meta-analysis.

Author 3 - Yang Xie evaluate the methodological quality and evidence quality, and revise the manuscript.

Author 4 - Jiansheng Li conceive this study. 